\title{
Carnets
}

Revue électronique d'études françaises de l'APEF

Deuxième série - $10 \mid 2017$

Pratiques de l'errance, vécus de la mémoire

\section{Errance et passage à la limite}

Avec Cormac McCarthy et Jean-Marie Gustave Le Clézio

\section{Éric Fougère}

\section{(2) OpenEdition}

Journals

Édition électronique

URL : http://journals.openedition.org/carnets/2230

DOI : $10.4000 /$ carnets.2230

ISSN : 1646-7698

Éditeur

APEF

Référence électronique

Éric Fougère, «Errance et passage à la limite », Carnets [En ligne], Deuxième série - 10 | 2017, mis en ligne le 30 avril 2017, consulté le 05 mai 2019. URL : http://journals.openedition.org/carnets/2230 ; DOI : 10.4000/carnets.2230

Ce document a été généré automatiquement le 5 mai 2019.

\section{(c) (i) \&}

Carnets est mis à disposition selon les termes de la licence Creative Commons - Atribution - Pas d'utilisation commerciale 4.0 International. 


\title{
Errance et passage à la limite
}

\author{
Avec Cormac McCarthy et Jean-Marie Gustave Le Clézio
}

\author{
Éric Fougère
}

1 La frontière est ce qui sépare et relie deux côtés dont elle participe à la fois. Ligne de partage, elle est clôture au-delà de laquelle on devient un étranger sorti du pays, mais aussi seuil à partir duquel on se laisse habiter par des paysages, aux confins d'un inconnu dont l'étrangeté paraît repousser toute limite. On parlera dans un cas d'errance, et dans l'autre on parlera plutôt de passage. Ici, mouvement désorienté dans les marges, et là, carrefour à la croisée de marches inventées par des chemins qui marquent. Un espace est tantôt celui du point tantôt celui du pont. Le point sera celui d'une île de quarantaine où, chez Le Clézio, des migrants sont internés dans l'attente de leur embarquement pour l'île Maurice. Et le pont sera celui qu'empruntent les personnages de McCarthy pour franchir la frontière américano-mexicaine. Une aventure existentielle attend les voyageurs. Au lieu du but affiché : retour à des origines incertaines au contact d'un ailleurs, on touche à des limites où le dépassement de celles-ci révèle un envers intérieur. Et la frontière, opérant normalement la territorialisation des lieux, finit par déterritorialiser l'espace en amenant l'homme au bout de lui-même et des choses.

2 La frontière américano-mexicaine est la matérialisation d'une limite abstraite intérieure à tout l'espace américain, de part et d'autre d'un seul et même fleuve en double appellation (Rio Grande/Rio Bravo), cher au western. Une Frontière majuscule est refoulée jusqu'à l'océan, bordure ultime et naturelle où se noie cet objet géographique instable à la dissolution duquel on doit l'apparition d'un double objet topique appelé border, au sens de frontière qui barre la route, ou frontier, au sens de front pionnier qui trace une voie. Dans un cas l'espace est fixé par des limites et dans l'autre il est sans arrêt déplacé. Le Sud est un substitut de l'Ouest absenté ${ }^{1}$, dont McCarthy ressuscite et simultanément démolit le mythe en 1985 avec Méridien de sang. C'est au Sud, à présent, que les cow-boys adolescents de la Trilogie des confins s'en vont prendre la route et perdre le nord et la vie, comme il arrive aussi dans No Country For Old Men en 2005. À chaque fois, passer la frontière équivaut donc à dépasser les limites. Un dépassement fait se poser la question du passage à la limite. Autant la frontière est ce qui territorialise un espace, autant son passage est ce qui désoriente un réel. On essaiera de montrer qu'il y va d'une inversion 
dont les deux côtés rabattus s'annulent en faisant le vide, en laissant place à l'absence, à l'errance. Il n'y a pas de retour à l'endroit. Seul est le sans-lieu. Seul est ce qui s'en va. Ce qui s'enlève et dérive.

3 La même errance, emblématisée par une rencontre avec Arthur Rimbaud lors d'une escale à Aden, est le résultat d'un déracinement comparable dans le roman de Le Clézio La Quarantaine (1995). La même espèce de dualité structure aussi cette errance, en redoublant toutefois le déracinement par un confinement, l'île de quarantaine par une frontière intérieure qui divise une île elle-même en relation de miroir avec une autre plus petite et qui sert en même temps de repoussoir. Et c'est là que la comparaison des deux romanciers diffère, en apparence. Autant l'espace américain se révèle ouvert, où la frontière est sans prise, en définitive ; autant l'espace indianocéanique, insulaire et non plus continental, est resserré, prisonnier. Chez McCarthy, l'errance est non-retour, alors que chez Le Clézio, les personnages, aussi défamiliarisés soient-ils, essaient tout au long de retrouver la mémoire de leur origine aux destins croisés, dans une écriture du contrepoint qui remonte au Le Clézio du roman Désert. Un jeu savant sur les initiales et les anagrammes y plaide en faveur d'une réversibilité de l'exil en promesse (au-delà de l'insularité forcée) d'un enracinement dans le monde après acceptation des limites insulaires et de l'illimitation maritime. L'épreuve de la mort (symbolique ou réelle) est gage de résurrection, quand elle ressemble à du néant, chez McCarthy.

\section{McCarthy : frontière et désert}

4 Le premier volet de la Trilogie commence avec un train surgi de l'est, alors que l'obscurité déchirée par un faisceau de phares est refermée sur une "interminable clôture " (McCarthy, 1998:10) et que l'espace entrevu se mesure au nombre de kilomètres indiqués par des poteaux, de distance en distance, au plus loin de l'époque et de l'espace où des Indiens chevauchaient «sur les plaines du Sud» (idem: 13), en direction du Mexique. Au lieu des pistes anciennes, une voie ferrée. Plutôt que des traces, un tracé rectiligne. John Grady Cole est orphelin. Son père est mort. Avec lui tout un monde a disparu : celui de « si jolis chevaux » qu'on ne voit plus que le long de chaussées martelées par les semiremorques ou par les chevalets de pompage et ponctuées des débris de moulins à vent pareils à ceux de Don Quichotte. Un monde anachronique où rien ne sera plus jamais... pareil. «Est-ce que tu connais le pays de l'autre côté ?» (idem: 42) demande un des adolescents partis vers l'ouest et prenant vers le sud au Mexicain croisé sur la route. «Y a rien marqué de l'autre côté » (ibidem). De ce côté-ci : des routes et des cours d'eau, des villes et des collines : une géographie. De l'autre côté du Rio Grande : le blanc de la carte et l'inconnu d'un ailleurs absolu - du pur espace.

Un désert est, dans Méridien de sang, ce qui se présente à qui franchit les limites et consent à l'espace. Il est sans fin. Le premier effet du passage à la limite est l'illimitation. Le deuxième est la sauvagerie barbare où quarante-six cavaliers d'apocalypse errent en diminuant, comme des somnambules ou des fantômes aux marges de la Création, sous un soleil ténébreux, sur une route d'enfer. Un troisième effet, résultant des deux précédents, consiste à déréaliser l'être au profit d'un monde élémentaire et minimal, impersonnel et $\mathrm{nu}$ : des traînées de nuages et de poussière, un trou de soleil, une ombre de monde, une terre gaste aux marches du plus-rien. Géographie réduite à la géologie d'un ossuaire minéral usé par le vent. Espace amené par le vide à son étale et létale évidence. Interroger cette évidence est possible en situation frontalière et ne l'est pas dans l'espace institué 
d'un désert où l'ubiquité des choses et leur unanime égalité défont le réel et défient la pensée jusqu'à l'indifférence. Il faut changer de ligne et revenir à la frontière comme à ce qui restaure une dualité par-delà le règne indéterminé du neutre.

6 Dans Le Grand Passage, un loup qui s'attaque au bétail, arrivé des montagnes, est capturé par Billy Parham et reconduit vers le Mexique. Et c'est pendant cette opération que les parents de Billy sont tués par un Indien que lui-même et son frère avaient secouru. L'Amérique antérieure est sans frontière. Un loup la parcourt en tous sens. Un Indien la figure en errant comme un loup. Le fils du ranch américain, lui, ne peut la rencontrer qu'en traversant le fleuve à la fois funèbre et baptismal, en même temps Styx et Jourdain, séparant les deux côtés du temps. La frontière internationale existe à peine avec ses bornes en béton. Le loup suit déjà comme un chien son convoyeur à cheval. On ne s'aperçoit pas du passage. Il est midi. C'est l'heure où le médian coagule et suspend l'espace à l'abstraction du mitan. Tout autre est la vraie frontière. Autre est le « grand passage ». C'est quand Billy fait passer cheval et loup de l'autre côté du cours d'eau qui leur barre un peu plus loin le chemin que les ennuis commencent. Ils commencent avec le tribut d'identité qu'un Charon lui demande et qu'il ne peut donner puisqu'il est sans papiers. L'identité vacille. Il n'y a pas de titre à la propriété de la nature incarnée par le loup. La mort est embarquée du côté choisi par la direction prise.

\section{La matrice : un monde autour, un monde au fond}

7 Un vieux Mexicain mourant consulté par Billy sur les pièges à loups parle de matriz en espagnol. La matrice est naturellement l'organe du loup femelle dont l'odeur est susceptible d'attirer tous les loups mâles. Elle est difficile à définir. Elle signale aussi bien ces confins du monde où les actions de l'homme et de Dieu se confondent. Il n'est pas question de découvrir un tel point mais plutôt de le reconnaitre au moment de son apparition, dit le vieillard. Or il n'y a plus de loups selon le vieux Mexicain. Tous ont été tués. Comme les bisons de Méridien de sang. C'est donc à sa disparition qu'on connaîtra le lieu du lien mentionné. On dira que cet endroit, s'il existe et si ce n'est pas son envers, est celui d'un monde qui brûle « au bord d'un vide inconnaissable » (McCarthy, $2000: 86$ ), à l'image des yeux du loup perçant l'obscurité « comme des lampes aux portes d'un autre monde » (ibidem). On dira que la matrice a quelque chose à voir avec la frontière, à condition de distinguer son acception géographique et son sens existentiel.

Il y a deux spatialités. L'une est celle du «monde autour » et l'autre est celle du « monde au fond ", comme il est dit dans De si jolis chevaux (McCarthy, 1998: 71). Dans Le Grand Passage, un des nombreux personnages de rencontre à qui le discours de sagesse est spécialement dévolu communique une prophétie de mort à Billy pour enjoindre à celui-ci de ne plus errer, « car si le monde semble être un lieu où résident les hommes c'est dans l'homme en réalité que réside le monde et pour le connaître c'est (...) là qu'il faut chercher » (McCarthy, 2000 : 152). Colindancia désigne encore en espagnol une idée de " pacte de mitoyenneté » que la théologie d'un prêtre émissaire entend faire admettre au solitaire enfermé dans l'église d'un village entièrement détruit par un tremblement de terre et qui, révolté contre la Création, n'en finit pas de blasphémer. Tirer des lignes et fixer des limites entre le monde et Dieu serait une façon de réconcilier l'homme et la Création. Mais les deux ne font qu'un même ici dehors où la frontière en même temps se réalise et s'abolit. 
«J'peux pas rester dehors là-dedans » (McCarthy, 1998 : 78) dit le plus jeune adolescent de De si jolis chevaux, n'ignorant pas que la réalité qu'il désigne aussi contradictoirement sous l'orage est le destin qui l'a déjà désigné pour être foudroyé malgré toutes les précautions du garçon qui va mourir et qui le sait. "Mais il faut que je me mette quelque part » ( ibidem) a-t-il beau répéter, rien n'y fait : « la mort est la vérité » (McCarthy, 2000 : 431). Une évidence est cette "obscurité du dehors" (outer dark), qui donne son titre à un roman de McCarthy de 1968 et va de pair avec l'obscurité du dedans scellée par la confusion de l'homme et du monde au plus profond de l'obscurité du cœur de la terre. Un roman récent de McCarthy signifie bien ces deux côtés de toute chose. Un tueur y joue la vie des autres à pile ou face. On croit son jeu sans queue ni tête. Il est à la poursuite d'un fugitif en même temps recherché par un shérif. Ils sont doublés par un groupe mexicain de passeurs de drogue eux-mêmes aux trousses du voleur en cavale et par un groupe américain d'acheteurs spoliés dans le marché passé contre argent pour avoir la drogue. On a reconnu le canevas de No Country For Old Men, où se poursuit la substitution de la ville au désert entamée dans Des villes dans la plaine en 1998.

Il faut être prêt à mourir. Il a toujours fallu l'être, assure le shérif au début du roman. La nouveauté, c'est qu'il faudrait maintenant jouer son âme. Ainsi posée, la question devient métaphysique et c'est sur la question de réversibilité du mal et du bien que la notion de frontière est relancée. La même infortune arrive aux victimes de la frontière chez McCarthy. C'est en voulant faire le bien qu'ils font leur malheur. John Grady Cole pour ne pas abandonner Blevins à ses cauchemars et à ses poursuivants mexicains. Billy Parham, on l'a vu, pour ne pas abandonner la louve qu'il a prise au piège à la mort. Et le Moss de No Country For old Men pour avoir eu pitié de l'agonie d'un mafieux brûlé par la soif et terrorisé par la peur des loups. Mais il est vrai de dire aussi que de la mort et du mal il peut sortir un bien. John et Billy réunis dans le dernier volet de la Trilogie des confins s'en vont chasser des chiens prédateurs avec une cruauté qui ne va pas jusqu'à ne pas recueillir un chiot de la meute exterminée.

\section{La soudure ou l'embrouille?}

Moss est le voleur inopiné d'un argent sale. Il exerce aussi la profession de soudeur. Il est quelqu'un pour qui le raccord existe avec un génie du bricolage égal à celui de l'engin de mort assemblé par l'assassin Chigurh au moyen d'un pistolet d'abattoir et d'une de ces bouteilles d'oxygène en usage dans les hôpitaux pour pallier les insuffisances respiratoires. Un transpondeur est ce qui relie l'un à l'autre à distance. Ils sont tous deux sur la corde raide, entre le mal et le bien pour le premier, entre la vie et la mort pour les deux, ne tenant plus qu'au fil d'une frontière de plus en plus ténue. La séparation des deux côtés n'est pas étanche. Il y a toujours un peu de la chèvre et du chou dans les histoires de frontière et de fleuve à traverser. C'est du moins la vision qu'en a le shérif Bell, adepte du compromis. Mais celui-ci ne veut pas jouer son âme. Il n'a pas d'action sur la réalité d'un monde aiguisé comme une lame de rasoir au tourniquet de la douane et des changeurs de monnaie qui ne font que donner le change et le tournis. Le nouveau monde est celui de l'embrouille. Il n'est plus celui des valeurs. Il est celui du trafic et n'est plus celui de l'échange. Il faut laisser de côté la soudure. Une indifférenciation généralisée menace la frontière des villes comme elle minait le désert alentour. $\mathrm{Y}$ a-t-il encore une frontière ? Il y a deux groupes aux basques de Moss et peu importe lequel c'est, puisque ce n'est évidemment pas l'autre, et que l'autre est encore moins le même. 
12 À l'indifférenciation dans laquelle une réversibilité dissout la dualité du réel il faut opposer la vérité noire et nue. «Je crois la vérité (...) toujours simple » (McCarthy, 2007 : 236) assure le shérif. "Il y a le même nom sur la liste de tout le monde. Et c'est le seul nom qu'il y a dessus » (idem: 149). Cette vérité tranche. Elle ne découpe pas le monde. Elle coupe. Évidente, elle est simple. Indivise, elle est unique. Il n'y a pas d'espace où l'on pourrait s'en cacher. Parler de frontière a du sens à condition d'y voir alors un symbole. En repassant en taxi sur un pont qui doit le reconduire à la limite où l'argent volé se trouve enfoui sous des joncs bordant le cours d'eau, Moss prend cinq billets de cent dollars et les déchire à l'intention du chauffeur en deux moitiés pour acompte, et cette opération même est ce qu'on entend par frontière au sens de coupure. On comprend ce qu'est la vérité d'évidence imagée par la frontière. Il y a deux moitiés de toute chose et l'on ne revient pas de l'autre côté du monde ou de la vie pour les réunir. Elles sont à jamais solidaires et scindées. Une figure insistante en est le chiasme : «Au fond de toi tu l'es. Tu l'es au fond de toi » (McCarthy, 1998: 19), dit le fils au père qui doute d'être resté le même. Moss offre cinq cents dollars à des garçons pour un blouson qui doit dissimuler le sang de ses blessures. Il doit marchander. D'autres garçons donnent pour rien leur chemise à Chigurh afin qu'il en fasse une écharpe à son bras disloqué. Chigurh insiste pour leur donner l'argent qu'ils refusent. On ne veut pas de l'argent du tueur. On réclame à la victime aux abois le sien. Chiasme.

13 Une autre figure est la tautologie. De Moss sa jeune épouse dit qu'il est ce qu'il est (McCarthy, 2007 : 123). À la jeune fille prise par Moss en stop, il est dit qu'il arrive les choses qui arrivent (idem : 230). Elles sont comme elles sont, dit Chigurh (idem : 246). C'est l'évidence. Il y a bien deux moitiés des choses et deux côtés de la frontière en miroir. On ne peut que les refléter les uns dans les autres. Ils ne sont rien par eux-mêmes et séparément. Leur unique raison d'être est la relation de stricte identité qu'ils instaurent en deux parts égales et répétées. L'évidence est la révélation d'un double secret: le vide est la vérité. Le passage est la disparition.

Ce n'est pas que toute vérité soit creuse. C'est que tout espace ouvert en elle est vide. Le vide est l'espace de l'évidence. "Y a pas de milieu », dit John Grady Cole à son ami Rawlins en prison. «Les laisse surtout pas croire qu'ils auront pas besoin de nous tuer. (...) J'ai bien l'intention de faire ce qu'il faut pour qu'ils essaient d'avoir ma peau. (...) Ou bien ils nous font la peau ou ils nous foutent la paix. Y a pas de milieu.» (McCarthy, 1998: 206-207) C'est au milieu du pont séparant la frontière en deux qu'est pourtant cachée la valise pleine d'argent de Moss. Le milieu serait l'emplacement d'un espace où sont fixées des limites une fois pour toutes. Or le fleuve au bord duquel est la mallette ne cessera de couler. « Y a pas de limites » (McCarthy, $2007: 214$ ) enchérit Moss. Il n'y en a que pour le shérif Bell, et dans un «monde qui recule » (idem: 113). Il n'y a pas non plus d'alternative. John Grady tue pour sauver sa peau dans un volume et meurt à la fin d'un autre avec le même couteau. "Je sais comment cela ne finira pas" (idem: 125) dit le shérif. Mais: "Vous savez comment ça finira» (idem: 175) dit en écho Chigurh. Un "démon de l'identité » qui, selon Clément Rosset (Rosset, 2008 : 309-351), se trouve au fondement du réel, interdit de penser la frontière autrement que comme un lieu du lien déchiré, là même où des fonctionnaires de police s'engraissent avec l'argent d'une drogue vendue à des enfants qui l'achètent. Où commerce est devenu l'autre nom de guerre. 


\section{Le Clézio : la part double, entre déracinement et confinement}

La Quarantaine est un roman du déracinement. Tous les personnages y sont des exilés quand ce n'est pas, comme la Giribala d'un des récits emboîtés, des parias. Les deux frères Jacques et Léon n'ont de cesse qu'ils ne retournent à l'île Maurice, où leur mère, une Eurasienne adoptée par un Anglais mais née en Inde, incarne la part étrangère au monde européen de la même façon que les ascendants mexicains de Billy représente elle aussi l'autre côté des États-Unis chez McCarthy, dont les romans de la Trilogie sont émaillés de phrases en espagnol comme le roman de Le Clézio lui-même est imprégné de vocables en référence à l'Inde. Et, de la même façon que John Grady Cole et Billy tombent amoureux de Mexicaines, Léon coupe avec l'Europe en choisissant de rester avec Suryavati, jeune Indienne dont la mère est née d'une Anglaise adoptée par une Indienne, à l'occasion des mêmes événements historiques (la grande mutinerie des Cipayes) qui font qu'Amalia, mère de Léon, et Ananta, mère de Suryavati, non seulement s'apparentent en paronymie mais frayent aussi la voie d'un passage, en métissage.

Un premier passage est celui qui s'effectue sur l'île de quarantaine où les passagers d'origine européenne à destination de Maurice (un couple anglais formé de John et de Sarah, le couple constitué par Jacques et Suzanne, et celui qui fait se rapprocher deux personnages antipathiques: Bartoli et Véran, dit Véreux), rejoignent des migrants indiens coolies sous contrat de main-d'œuvre. Autant les deux premiers couples ont une identité marquée, soulignée par identité de leurs initiales, autant celui qui va s'établir entre Léon et Suryavati, durant le séjour obligé sur l'île Plate, appelle un franchissement de la barrière ethnique. Il est matérialisé par la transgression de la frontière instaurée de part et d'autre des deux côtés de l'île: un côté pour les Indiens, que sépare encore une limite intérieure (entre immigrants et parias), un côté pour les Européens. Les premiers sont regroupés dans des huttes, et les seconds sont retirés dans les bâtiments de la Quarantaine « en dur ». Il n'y a que Léon pour errer dans l'île, à la poursuite de Suryavati, qui rôde elle-même à la recherche d'ourites, en bord de mer.

On voit s'opposer deux insularités. L'insularité sanitaire est d'inspiration panoptique. Une lunette d'approche est constamment braquée par Véran sur le camp des Indiens depuis les hauteurs du relief où se dresse un phare. C'est l'insularité du confinement forcé. L'insularité de l'assignation disciplinaire. Une insularité rivale est l'insularité des confins, spatialisée par la plage (et non plus la crête). Or la plage est un espace ambivalent. Comme bord, elle constitue certes une limite, et c'est bien de celle-ci que les isolés sont prisonniers ; mais, comme bord ouvert à la mer, elle est aussi le lieu des confins que la mer illimite. Autant la frontière établie par la surveillance est une ligne de démarcation très théorique, autant la bordure littorale est ici le point concret de fixation d'une aspiration contradictoire, à l'instar du mouvement des marées qui la couvrent et la découvrent : orientée vers un besoin d'évasion (car l'île Maurice, au large, est en vue), la plage est aussi cet espace intermédiaire où le récif, en la rédupliquant, forme et ferme une mer intérieure, à distance égale entre les îles Plate et Gabriel.

Une axiologie fait valoir horizontalité pélagienne (en ligne de fuite) et profondeur lagunaire (en point d'ancrage). Il s'agit, pour Léon, d'habiter cet entre-deux liquide en enfonçant dans les racines alternées du monde (on sait que, par une passe, chaque marée fait se remplir et se vider le lagon, qui devient quasi sec à marée basse). Il importe à 
Suryavati, qu'on voit pêcher sur «l'arc des récifs » (Le Clézio, 1995 : 75), de décider de suivre Léon sur l'îlot Gabriel, une fois fait par les autorités le choix de surinsulariser les malades européens. Nous assistons donc au chassé-croisé de figures à la fois liantes et déliantes. Le lagon relie Plate et Gabriel à la façon dont y sont liés les deux personnages à leur amour insulaire. Mais les bûchers sur la plage où sont brûlés les morts, du côté du village des parias, s'ils constituent pour Léon le lieu d'un passage initiatique à la culture indienne, et le rapprochent ainsi de ses origines maternelles, assurent une fonction de déliaison funèbre qu'on ne peut s'empêcher de voir encore à l'œuvre avec les allersretours effectués par le vieux nautonier passeur ${ }^{2}$ entre Plate et Gabriel. Or, et c'est là tout l'intérêt de Le Clézio, les deux fonctions se retournent: il n'est pas de véritable attachement sans détachement. C'est seulement quand sa mère aura brûlé sur un bûcher que Suryavati rejoindra Léon sur l'îlot Gabriel. Et c'est au prix de la séparation de Léon d'avec Jacques et Suzanne et de sa disparition sans laisser de traces dans la colonie que s'accomplira pour lui le passage à la limite.

\section{Enracinement dans les confins}

19 La réversibilité de toute frontière, en tant que limes (ou limite) et limen (ou seuil), est étayée, comme on l'a dit, sur la réalité de deux côtés présents l'un à l'autre et pourtant séparés. Ces deux côtés sont bien représentés par un dernier personnage, Anna, dont le nom se lit justement comme un palindrome (au même titre qu'Ava, nom du bateau transportant ses passagers vers Maurice) et se trouve être en même temps le nom du domaine, à Maurice, où sont nés Jacques et Léon. C'est une vieille dame ayant vendu ce domaine quand le petit-neveu de Léon vient la voir en touriste à Maurice. Hormis le fait que l'aristocratie créole et les préjugés de caste ou de classe ont fait rompre Anna, jeune, avec une amie d'origine indienne, un point retient l'attention dans la dernière partie du roman : la vieille a pour occupation d'empoisonner les chiens. C'était l'occupation de Rimbaud malade au début du livre. Au-delà de ce parallèle, on ne peut manquer d'en faire un autre en lisant ce que dit Léon de son expérience insulaire en quarantaine : «J'étais un autre » (idem : 275), écho de la formule archi-connue du Voyant. Le dépouillement de ses liens d'appartenance imaginaire à Maurice a fait chez Léon ce que le « dérèglement de tous les sens » a fait chez le poète. Or "[i]l n'y a plus de poésie » (idem: 350), car après l'îlot Gabriel où les deux amoureux vivent au creux d'une faille habitée par un peuple d'oiseaux, « [i]l ne peut rien y avoir (...), seulement ces rochers noirs, nus et âpres, le vent qui siffle dans les buissons, la mer qui cogne. Rien d'autre que le basalte, la poussière, la cendre. Et le ciel où fusent les nuages, scellés sur les étoiles, et les pailles-en-queue dans les tanières, leur œil sans paupière qui attend le soleil » (idem : 377 ).

On comprend que l'errance insulaire, au mépris des frontières (spatiales), en défi des barrières (ethniques), arrive, " au bord de la terre, au bout du monde » (idem: 402), à substituer le secret d'un commencement total au mythe éventé d'une origine à Maurice : « Il me semble que j'ai vécu toute ma vie sur Plate, c'est ma terre natale, c'est là que j'ai tout appris, il n'y avait rien auparavant, il n'y aura rien après » (idem : 292). Si la leçon ne consistait que dans cette relocalisation, fût-ce au prix d'un abandon du personnage au Cosmos, on trahirait ce qui définit l'errance et la frontière. Errance : à l'exemple de Rimbaud, qualifié de "voyageur sans fin", qui donne son titre à la première partie du roman ; frontière : à l'image d'Anna, dont le nom se lit dans les deux sens. Or le voyageur est cloué par sa jambe au lit d'un hôpital d'Aden en début de roman. La vieille Anna 
liquide, en même temps que la propriété familiale, un passé que son neveu voudrait par contre hériter dans la dernière partie du roman mais qui ne reçoit d'Anna qu'un cahier qui nous ramène à l'ambiguïté de toute frontière : en rupture, en suture.

Il reste à méditer "J'étais un autre ». On y lira peut-être une traduction de l'errance en tant qu'il y a dissociation du sujet (verbal ou philosophique) et de l'attribut (grammatical ou psychologique) au sein d'une relation d'identité qui revient à se maintenir en se niant. Mais on analysera surtout l'énoncé comme une exemplification du concept de frontière: deux entités, distinctes a priori, sont soudées par la copule au-delà de la "cassure " initiale : «Elle m'a été donnée à la naissance, comme une marque, comme un goût de vengeance. Lorsque mon père a quitté la maison d'Anna, l'année de ses douze ans, l'ancienne brisure est entrée en lui, elle s'est continuée, elle s'est propagée d'année en année, jusqu'à moi » (idem : 457). On pourra voir, enfin, l'idée d'un passage à la limite : un autre, mais lequel? un autre quoi? Ce n'est ni simple identité ni pure altérité, mais quelque chose où les deux flottent, en devenir. On reconnaît dans ce devenir un souhait d'enracinement dans les confins qui fait dire à Léon 2 (le petit-neveu) : «Alors je suis devenu Léon, celui qui disparait, celui qui tourne le dos au monde, dans l'espoir de revenir un jour et de jouir de la ruine de ceux qui l'ont banni. Comme Léon dans la pension glacée de Rueil-Malmaison, je rêve de la mer éblouissante, du bruit de la mer sur les rochers noirs d'Anna. Un jour je reviendrai, et tout sera un à nouveau, comme si le temps n'était pas passé » (ibidem).

L'identité, contrariée par un être-autre, est maintenant déliée par une identification qui fait place au devenir-même (Léon 1 et Léon 2 confondus), dans l'unité d'une autreté recomposée : «Je reviendrai, et ce ne sera pas pour posséder la fortune des sucriers, ni la terre. Ce sera pour réunir ce qui a été séparé, les deux frères, Jacques et Léon, et à nouveau en moi, les deux ancêtres indissociables, l'Indien et le Breton, le terrien et le nomade, mes alliés vivant dans mon sang, toute la force et tout l'amour dont ils étaient capables » (ibidem). Autreté: le terme (otherness) est traduit de Homi Bhabha (The Location of Culture, 1994) par Bénédicte André, qui l'explique ainsi : «je suis moi parce que je ne suis pas Autre, mais je ne pourrais être moi sans la présence de l'Autre, en face de moi et en moi $^{3}$.» L'état d'identité figé dans sa perte est redéployé vers un processus identificatoire où les moitiés séparées sont unies dans ce qui continue de les disjoindre, à l'instar de toute frontière. Il n'y a pas de synthèse dialectique au bout de ce processus. Et si le «J'étais un autre » advient pour désigner le résultat du passage du corps amoureux de Léon dans celui de Suryavati ${ }^{4}$, ce n'est pas pour « être quelqu'un d'autre » (je souligne, idem: 274) mais pour devenir cela qui fait de l'acte amoureux la métaphore du socle insulaire («C'est le mouvement de la mer qui ronge et cogne le socle de l'île », idem : 376).

\section{Conclusion}

Un faux juge est aux prises avec un ex-prêtre. Ils se disputent la vie du "gamin » de Méridien de sang. Le même face-à-face de la morale et de la loi, des hommes et de la Création, se joue dans De si jolis chevaux. L'ex-prêtre est devenu révérend. Le faux juge est devenu vrai. Bon, dit l'un. Bien, dit l'autre. On pourrait penser qu'une inversion d'axiologie s'est produite entre les deux romans. Que la morale et la loi se sont ressoudées sur fond de guerre (au Mexique, en Europe, au Vietnam, en Corée), mais, entre les deux, le shérif hésite encore : observer la morale ou suivre la loi ? Pour qui pense avoir bien lu McCarthy la question reste en suspens, voire en tension. La frontière est poreuse. On y 
parle en espagnol et en anglais d'Indiens sans curiosité qui regardent une ombre jumelée de cheval et de cavalier passer, qui la regardent uniquement parce qu'elle passe et disparaît comme une seule et même créature en s'enfonçant dans le désert aux dernières lignes de De si jolis chevaux. Passer la frontière américaine en direction du Mexique introduit donc au passé d'une Révolution dont le sens est perdu dans un désert où rien n'échappe à la vérité du vide. Il faut avoir passé la limite au-delà de toute raison pour jouer son âme et risquer son existence à la conquête d'une vérité dont le principe est qu'on en meurt et dont le fin mot retombe en énigme. On ne revient pas de l'autre côté.

«Du plus loin qu'elle se souvenait, Ananta n'avait jamais arrêté de bouger, de fuir, d'attendre des bateaux, de marcher sur les routes. Maintenant elle ne voulait plus entendre cet homme qui appelait les noms, elle ne voulait plus monter dans le bateau, aller dans ce pays, Mirich Desh [Maurice], cette île d'où personne ne revenait » (Le Clézio, 1995 : 372). Léon renonce au monde en rêvant d'un retour à l'île Maurice à laquelle il va tourner le dos pour faire face à la vérité qui l'attend quand son errance en deçà des frontières de l'île Plate a pris fin, quand il est passé du côté des parias, de ceux dont ne veut personne : Indiens de l'autre côté de l'île, Européens malades et bientôt morts à l'îlot Gabriel. Ananta n'a de cesse qu'elle ne fasse défiler par Léon les noms, les images et les sensations qui pourraient tant soit peu rappeler ce que fut sa prime enfance anglaise. Aux deux bouts de la généalogie tortueuse et tourmentée des personnages, en sens inverse et symétrique, une mémoire improbable enchaine à des origines inaccessibles où rien n'est possible, hormis leur abandon. Car on ne revient pas dans le passé vécu par procuration des autres. Il y a bien continuité, mais elle est sans solution malgré les frontières établies pour forcer le passage. Un seul est le bon, qui fait du non-retour une condition de dépossession souveraine : «Quand j'ai compris cela, qu'elle [Suryavati] n'avait plus rien, j'ai senti un frisson, le frisson que donne la proximité de la vérité » (idem : 346$)$.

\section{BIBLIOGRAPHIE}

ANDRÉ, Bénédicte (2016). Illéité, perspectives littéraires sur le vécu insulaire. Paris : Pétra.

LE CLÉZIO, Jean-Marie Gustave (1995). La Quarantaine. Paris : Gallimard.

MCCARTHY, Cormac (1998). De si jolis chevaux (All the Pretty Horses, 1992). Paris : Actes Sud, Points Seuil.

MCCARTHY, Cormac (2000). Le Grand Passage (The Crossing, 1994). Paris : L'Olivier, Points Seuil.

MCCARTHY, Cormac (2007). No Country For Old Men (2005). Paris : L'Olivier, Points Seuil.

ROSSET, Clément (2008). L'École du réel. Paris : Minuit. 


\section{NOTES}

1. Voir Anne Besson, «De part et d'autre de la frontière : The Border Trilogy de Cormac McCarthy (1992-1998), La Frontera de Cristal de Carlos Fuentes (1995) », in Yves Clavaron et Bernard Dieterle dir., Métissages littéraires, Actes du XxxII ${ }^{\mathrm{e}}$ Congrès de la Société Française de Littérature Générale et Comparée, Saint-Étienne 8-10 septembre 2004, Centre d'Études sur les Littératures Étrangères et Comparées, Centre d'Études Comparatistes, Publications de l'Université de Saint-Étienne, 2005, p. 42.

2. Il a son équivalent dans l'histoire emboîtée qui se déroule en Inde avec un Indien qui fait passer le fleuve à Giribala (la mère) et Ananta (la fille).

3. Bénédicte André, Îléité, perspectives littéraires sur le vécu insulaire, Paris, Pétra, 2016, p. 22. «Je choisis le néologisme afin de souligner la recherche lexicale de Bhabha et sa volontaire mise à distance de alterity (altérité) ou strangeness (étrangeté). Il est à noter qu'en 1936, Antonio Machado, forgeait le néologisme otredad (autreté) afin de désigner une hétérogénéité de l'être, une ouverture du sujet à l'Autre. Le concept sera repris par Octavio Paz : comme 'l'autreté est dans l'homme même', par la 'révélation poétique', Je deviens capable d'être un autre. Pas complètement inconnue dans une langue romane proche, l'autreté entretient donc bien des rapports avec la fracture du même par l'otherness, aux confluents de la parole et de l'éthique. » $\mathrm{L}$. Dubreuil, «Alter, inter : académisme et postcolonial studies ", Labyrinthe, vol. 2, n 24 (2006), p. 54. Cité par B. André, op. cit., p. 22.

4. "J'ai senti la houle de son corps contre moi. Sous sa peau les éclats endurcis du basalte, et la poussière, comme de la cendre. Le goût du sel sur ses paupières, le bruit du sang dans mes artères, dans sa poitrine. Je l'ai pénétrée et elle a tourné un peu de côté son visage, parce que je lui faisais mal. Mais le désir m'emportait, si vite que je ne pouvais m'arrêter, maintenant j'entendais son souffle, mêlé à mon souffle, je sentais son corps frais comme l'eau qui coule, j'étais devenu le feu, la fièvre, le sang, et Surya me serrait entre ses cuisses d'une étreinte puissante » (Le Clézio, 1995 : 274).

\section{RÉSUMÉS}

La frontière instaure une relation d'identité paradoxale : il y a deux côtés qui participent à la fois de ce qui sépare et de ce qui relie. Ces deux moitiés, rabattues, s'annulent et font place à l'errance : autant la frontière est en effet ce qui territorialise, autant son passage est ce qui désoriente. On distinguera cependant l'errance et le passage. Ici mouvement sans fin dans les marges, et là carrefour à la croisée de marques inventées par des chemins. Le passage implique un limen, où l'errance induit par défaut le limes. Un seuil est celui de l'île où, chez Le Clézio, des migrants sont internés pour être ensuite embarqués vers l'île Maurice. Une limite est le pont qu'empruntent les personnages de McCarthy pour franchir la frontière américano-mexicaine. $\mathrm{Au}$ lieu du but affiché (retour à des origines incertaines au contact d'un ailleurs), on touche à des limites où le dépassement révèle un envers intérieur amenant l'homme au bout de lui-même. Il n'y a pas de retour à l'endroit. Seul est ce qui s'enlève. 
Borders establish a paradoxical relationship of identity in that both sides created participate at the very same time in a process of separating and of connecting. Once folded onto one another, these two halves cancel each other out and make way for a wandering: as much as borders territorialise, their manner of passing through can disorientate. However, it is necessary to distinguish the experience of wandering (an endless movement within the margins) from that of passing through (a crossroad consisting in the many paths one may take). Passing through implies a limen (threshold), whereas wandering by default infers a limes (limit). In Le Clézio, the island is a threshold where migrants are detained before being shipped off to Mauritius. A limit is seen in the bridge that McCarthy's characters take to cross the American-Mexican border. Whilst it could be tempting to think that the contact with elsewhere necessarily translates into a return to an admittedly uncertain starting point, crossing over the limit (spatiality) reveals a flipside where characters confront their own limitations (ontology). Once the limit is crossed, return becomes impossible. What remains is that which drifts away.

\section{INDEX}

Mots-clés : déracinement, confins, confinement, passage, errance

Keywords : uprooting, limits, confinement, crossing, wandering

\section{AUTEUR}

\section{ÉRIC FOUGÈRE}

Université Blaise Pascal (Clermont-Ferrand)- Centre de recherche sur la littérature de voyage (CRLV)

eric.fougere98[at]gmail.com 